\title{
Les sections bilingues français - langue régionale et le cadre européen : nouvelles perspectives didactiques
}

Luc Bonet

\section{(2) OpenEdition}

Journals

Édition électronique

URL : http://journals.openedition.org/trema/435

DOI : $10.4000 /$ trema.435

ISSN : 2107-0997

Éditeur

Faculté d'Éducation de l'université de Montpellier

Édition imprimée

Date de publication : 1 septembre 2007

Pagination : 115 - 124

ISSN : 1167-315X

\section{Référence électronique}

Luc Bonet, «Les sections bilingues français - langue régionale et le cadre européen : nouvelles perspectives didactiques », Tréma [En ligne], 28 | 2007, mis en ligne le 04 octobre 2010, consulté le 30 avril 2019. URL : http://journals.openedition.org/trema/435 ; DOI : 10.4000/trema.435

Ce document a été généré automatiquement le 30 avril 2019.

Trema 
Les sections bilingues français - langue régionale et le cadre européen: nouvelles perspectives didactiques

Luc Bonet

I. Sections bilingues français - langue régionale et sections européennes de langues étrangères : comparaison des dispositifs 
1 Les sections bilingues français - langue régionale ${ }^{1}$ ont une claire antériorité dans l'Education Nationale par rapport aux sections européennes, l'autre modalité généralisable de l'enseignement des disciplines non linguistiques en langue seconde. La première mention officielle de la possibilité d'expérimenter l'enseignement bilingue français - langue régionale, «en tenant compte des expériences déjà engagées ", apparaît en 1982 dans la circulaire dite SAVARY ${ }^{2}$ portant sur l'enseignement des cultures et langues régionales dans le service public de l'Éducation Nationale. Les sections européennes ou de langues orientales qui concernent les langues étrangères n'ont été mises en place que dix ans plus tard, en 1992, afin de proposer au plus grand nombre d'élèves le modèle antérieur des

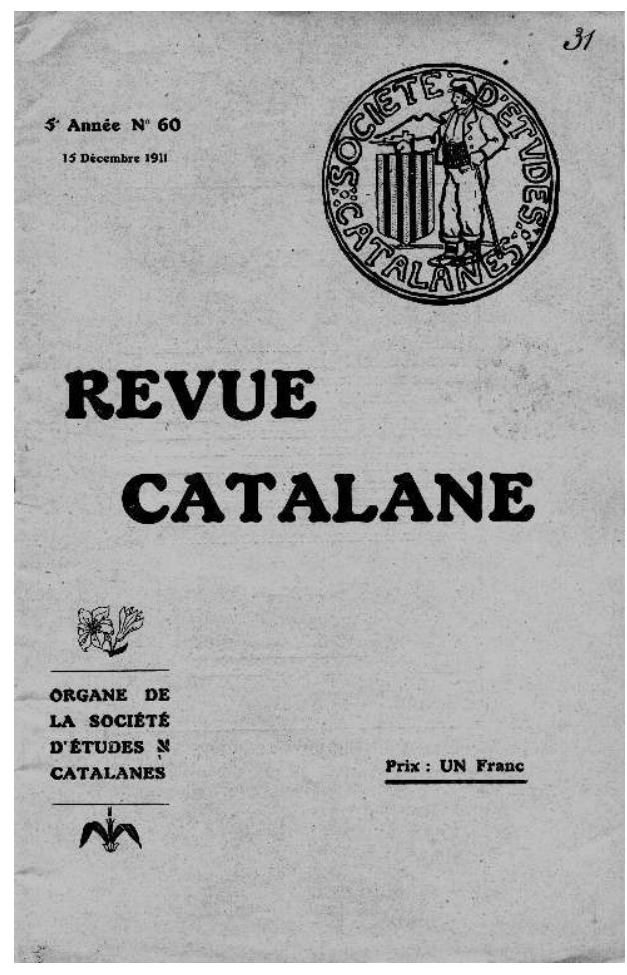
sections internationales qui existent depuis 1981, mais pour un public spécifique (au moins $25 \%$ d'élèves étrangers).

2 Les sections bilingues français - langue régionale se déploient de la maternelle à la terminale à la différence des sections européennes de langues étrangères qui ne sont implantées qu'à partir du collège, en général dès la classe de quatrième, grâce à un enseignement renforcé de langue étrangère, la DNL en langue seconde n'intervenant, dans la plupart des cas, qu'au lycée.

La circulaire dite BAYROU ${ }^{3}$ confirme effectivement en 1995 la mise en place d'un enseignement bilingue qui "commence dès le cycle 1 et se poursuit à l'école élémentaire. La langue régionale y est à la fois langue enseignée et langue d'enseignement ». "Le bilinguisme se définit par un enseignement à parité horaire entre la langue régionale et la langue française »4. Au collège, un enseignement d'une ou deux disciplines est proposé dans la langue régionale ${ }^{4}$. Le cursus bilingue français - langue régionale est prolongé officiellement jusqu'au lycée (général et professionnel) par la circulaire dite $\mathrm{LANG}^{5}$ de 2001 relative aux « Modalités de mise en œuvre de l'enseignement bilingue à parité horaire ».

3 La parité horaire entre les langues d'enseignement, instituée dès le premier degré et prévue pour le second degré, est donc aussi une spécificité des sections bilingues de langues régionales. Les sections européennes ne visent, en général à partir de la seconde, que l'enseignement de tout ou partie du programme d'une ou plusieurs DNL en langue étrangère, tandis que pour les sections bilingues le même dispositif relatif aux DNL est un moyen pour "atteindre progressivement un enseignement à parité en français et en langue régionale $"^{6}$ au collège et au lycée, dans la continuité de la parité horaire déjà installée à l'école primaire.

4 Cependant, cet enseignement pour moitié en français et pour moitié en langue régionale ne doit pas exclure la langue française de tout un champ disciplinaire, ainsi l'arrêté relatif à l'Enseignement bilingue en langues régionales à parité horaire dans les écoles, les collèges et les lycées, publié en 2003 sous le ministère FERRY7, précise : « Aucune discipline, 
ou aucun domaine disciplinaire, autre que la langue régionale, ne peut - être enseigné exclusivement en langue régionale ». Remarquons que cette précision n'apparaît pas explicitement dans les instructions officielles des sections européennes. En fait, voici une précaution réglementaire qui invite à une réflexion d'ordre didactique sur l'alternance linguistique en cours de DNL et qui permet de passer d'un bilinguisme reposant sur l'enseignement en langue seconde de certaines DNL, à un bilinguisme qui investit toutes les DNL. Nous y reviendrons.

5 La scolarité bilingue français - langue régionale fait l'objet d'une validation aux examens, au brevet et au baccalauréat. Dès 1994, un arrêté autorise ${ }^{8}$ "les élèves des classes de troisième des sections bilingues français - langue régionale [...] [à] composer en français ou en langue régionale lors de l'épreuve d'histoire - géographie du diplôme national du brevet ». Il est intéressant de relever que cette autorisation précède de 10 mois la publication de la circulaire de 1995 qui valide l'enseignement bilingue français - langue régionale à l'école et au collège, expérimenté depuis 12 ans en vertu de la circulaire de 1982, soit exactement le temps d'un cursus scolaire de la maternelle à la troisième. Dans le cas des sections bilingues de langues régionales, la réglementation relative aux examens précède celle qui organise ordinairement la scolarité, les pratiques dans les classes et la demande sociale en sont donc le moteur. De la même façon, la validation au baccalauréat des enseignements de DNL dispensés en langue régionale est mentionnée pour la première fois dans la circulaire de 2001, alors que les premiers candidats composent en langue régionale lors de l'épreuve d'histoire géographie en 1998, en langue catalane et de façon dérogatoire.

6 En revanche, les sections européennes ont disposé dès leur lancement en 1992 d'un cadre réglementaire complet. Cependant, la validation aux examens ne concerne que le baccalauréat lors duquel les candidats des sections européennes présentent une épreuve supplémentaire spécifique, ils bénéficient de la mention "section européenne " sur le diplôme. Les candidats des sections bilingues présentent en langue régionale, au brevet comme au baccalauréat, l'épreuve commune d'histoire - géographie, ils ne bénéficient d'aucune mention spécifique sur les diplômes, les rectorats délivrent parfois une attestation complémentaire.

7 Les modalités d'évaluation de la scolarité en section européenne révèlent une finalité d'ordre linguistique et culturel, le renforcement linguistique tardif, à partir de la quatrième, est le préalable à l'enseignement - apprentissage en langue seconde des DNL qui n'intervient qu'à partir de la seconde. Les élèves des sections européennes ne peuvent donc totaliser que trois années d'enseignement de DNL en langue seconde alors que les élèves des sections de langue régionale apprennent en deux langues tout au long de leur scolarité, la compétence linguistique en langue seconde ne précède pas la compétence notionnelle en langue seconde, elles se construisent mutuellement tout au long de la scolarité bilingue.

8 Les enseignants des DNL en langue régionale sont dans le second degré, soit des professeurs habilités à enseigner leur discipline en allemand (alsacien), basque, breton, catalan, corse ou occitan soit des professeurs de langue régionale ${ }^{9}$ dont la valence d'enseignement est l'histoire - géographie ou les mathématiques. Les professeurs d'école en section bilingue de langue régionale bénéficient d'une habilitation ou bien sont issus $\mathrm{du}$ Concours spécial de recrutement de professeurs des écoles en langues régionales ${ }^{10}$. Les enseignements de DNL en sections européennes (qui n'existent que dans le second degré) sont assurés par des professeurs habilités à enseigner en langue seconde ou par des enseignants d'histoire - géographie titulaires de la mention complémentaire «Langue 
vivante étrangère » depuis la session 2006 du CAPES. Les mentions complémentaires en langues régionales n'ont pas été créées.

9 Selon le site officiel Emilangues ${ }^{11}$, pour l'accompagnement des sections européennes et orientales, ces sections totalisaient, en 2006, 191655 élèves dont 63,4 \% inscrits en section anglais. Selon le site associatif FLAREP ${ }^{12}$, le nombre d'élèves scolarisés en section bilingue français - langue régionale s'élève, en 2006 - 2007, à 50311 élèves, dont $19 \%$ dans l'enseignement associatif. Les disparités d'une région à l'autre sont importantes, par exemple au Pays Basque (partie du département des Pyrénées - Atlantiques) $30 \%$ des élèves suivent une scolarité bilingue (la moitié dans l'enseignement public, un quart dans l'enseignement associatif et un quart dans l'enseignement confessionnel), en Catalogne ${ }^{13}$ (majeure partie du département des Pyrénées - Orientales), 3 \% des élèves sont en classe bilingue (les trois quarts à l'école publique et un quart dans les écoles associatives).

On vient de le percevoir, les sections bilingues de langues régionales ne sont pas le cadre privilégié officiellement pour le développement de l'enseignement des DNL en langues secondes même si, au niveau académique des politiques volontaristes en faveur des langues régionales sont mises en place par l'Education Nationale et / ou les collectivités territoriales. Dans l'académie de Montpellier, par exemple, le recteur Christian NIQUE a déployé à partir de mai 2006 un "Plan de développement de l'enseignement des langues régionales (occitan et catalan) $\aleph^{14}$, qui s'est traduit, parmi dix mesures concrètes, par l'augmentation du nombre de places au concours spécial de professeurs des écoles chargés de l'enseignement bilingue et l'édition d'une brochure ${ }^{15}$ sur le bilinguisme français - occitan et français - catalan.

\section{Enseignements linguistiques et disciplinaires décloisonnés pour sections bi - plurilingues français - langues régionales et étrangères}

11 Le développement des sections où les DNL sont enseignées en langue seconde est étroitement lié à la politique en faveur de ces mêmes langues; les sections bilingues de langues régionales en ont donc pâti, aujourd'hui de nouvelles perspectives didactiques se dessinent dans le sillage du Cadre Européen Commun de Référence (CECR).

12 Rappelons que le premier texte positif concernant l'enseignement des langues régionales est la loi dite DEIXONNE ${ }^{16}$ de 1951 qui en autorise un enseignement facultatif; ce n'est qu'en 1982, par la circulaire SAVARY, que les langues régionales deviennent «matière spécifique qui disposera donc de cadre horaire, de programmes, d'épreuves d'examen, de personnels formés et de programmes de recherche pédagogique est scientifique ». La loi de 1994 relative à l'emploi de la langue française, dite loi TOUBON, reconnaît l'usage des langues régionales dans l'enseignement. La loi d'orientation et de programme pour l'avenir de l'école de 2005, dite loi FILLON ${ }^{17}$, stipule qu' «Un enseignement de langues et cultures régionales peut être dispensé tout au long de la scolarité selon des modalités définies par voie de convention entre l'État et les collectivités territoriales où ces langues sont en usage ».

13 Les premiers programmes, très succincts, relatifs à l'enseignement des langues régionales n'ont finalement été publiés qu'en $1988^{18}$, dans le cadre des programmes du baccalauréat et ne concernaient donc que le lycée. Ensuite, à plusieurs reprises, notamment pour le collège en 1998 et 2002, des commissions d'experts ont été officiellement chargées de la rédaction de programmes; aucun projet n'a finalement été publié. Enfin, en 2003, les 
premiers programmes de langues étrangères ou régionales pour l'école primaire ${ }^{19}$ voient le jour, en vertu d'un possible enseignement de langues régionales, dès le cycle 1 , en parallèle avec l'enseignement obligatoire, dès le cycle 2, d'une langue étrangère. Dans le cadre de la réécriture de l'ensemble des programmes de langues vivantes consécutive à l'adoption en 2004 du CECR, les langues régionales ont été invitées en 2006 à une nouvelle rédaction des programmes du palier 1 (sixième et cinquième) du collège, niveau A220. Consécutivement, l'arrêté du ${ }^{20}$ mars 2007, publié au J.0. 81 du 5 avril 2007 fixe officiellement les programmes de l'enseignement de langues régionales au palier $1 \mathrm{du}$ collège en se référant à une publication en ligne ${ }^{21}$. La publication au B.O.E.N. du niveau A2, ainsi que des niveaux A1 de l'école primaire et du niveau B1 du palier 2 du collège ou du lycée écrits en 2007 devrait être effective avant la fin de l'année en cours.

La perspective didactique du CECR est dite " actionnelle », l'apprenant de langues devient un acteur social ayant à accomplir des tâches; la compétence visée est explicitement plurilingue et pluriculturelle. Il s'agit donc de « communiquer langagièrement et d'interagir culturelle - ment en tant qu'acteur social qui possède, à des degrés divers, la maîtrise de plusieurs langues et l'expérience de plusieurs cultures; il n'y a pas superposition ou juxtaposition de compétences distinctes, mais bien existence d'une compétence complexe, voire composite, dans laquelle l'utilisateur peut puiser $»^{22}$. "On ouvre des perspectives nouvelles pour fonder la discipline "langues vivantes" au - delà de la spécialité dans telle ou telle langue $»^{23}$ car jusqu'à présent, sauf exception, les apprenants " doivent gérer seuls cette construction, sur la base d'hypothèses et d'expériences plus ou moins heureuses, sans l'aide de leurs professeurs " ${ }^{24}$. C'est - à - dire qu'aujourd'hui, tout enseignement - apprentissage de langue devrait être mené de telle façon qu'il facilite l'apprentissage des autres langues; en retour, l'exploitation des interactions entre langues devrait faciliter l'apprentissage particulier de chaque langue.

Quel est donc l'écho de la compétence plurilingue, promue par le CECR, dans les programmes de langues vivantes de sixième et cinquième, les seuls permettant, à l'heure actuelle, une lecture commune aux langues étrangères et aux langues régionales? Dans le préambule commun aux programmes ${ }^{25}$ des collèges, palier1, la dimension plurilingue n'est considérée qu'en relation avec la langue française: "La réflexion sur les langues étrangères induit un retour sur le français et une prise de possession plus consciente des outils langagiers ». Dans le paragraphe consacré à l'écrit, on lit: «Les acquisitions du cours de français sont mises à profit, notamment en ce qui concerne le domaine de l'énonciation, pour aider les élèves à construire des stratégies de lecture des textes ». Aucune allusion n'est donc faite ni à l'exploitation des interactions entre les deux langues vivantes présentes dès le palier 1 du collège, en sections bi - langues (pour les langues étrangères) ou en sections bilingues (pour les langues régionales), ni à une éventuelle attention portée aux autres langues pratiquées par les élèves hors du cadre scolaire (langues de l'immigration par exemple).

Pour les langues étrangères les plus enseignées, les références à la compétence plurilingue dans les programmes sont inversement proportionnelles au degré de diffusion de la langue concernée dans le système éducatif. Ainsi, le programme d'anglais s'inscrit uniquement dans la prévention des interférences phonologiques avec le français: " Contrairement aux idées reçues, l'anglais n'est pas une langue facile pour les francophones". L'enseignement du portugais en revanche dépasse le cadre strict des interférences et interactions avec le français et s'inscrit dans la compétence linguistique complexe promue par le CECR et «contribut à la mise en place d'une conscience linguistique en relation avec l'apprentissage d'autres langues. » 
17 Parmi les langues régionales, les langues romanes sont aussi les plus enclines au travail de la compétence plurilingue. L'enseignement du corse "participe, aux côtés de celui du français, à la consolidation d'une compétence langagière globale. Il permet une meilleure approche des réalités corses, et ouvre à une dimension romane et méditerranéenne ». De même, l'enseignement de l'occitan - langue d'oc s'appuie « avec discernement sur les réalisations du français régional, de manière à faire prendre conscience des interactions entre les deux systèmes linguistiques de l'occitan et du français. On pourra également mettre en oeuvre avec profit diverses approches comparatives [...] avec d'autres langues romanes, notamment dans le cadre de "parcours romans" ". Dans la même direction, "l'enseignement apprentissage de la langue et de la culture catalanes, d'abord aux côtés du français mais aussi des langues étrangères, par le travail sur la propre altérité linguistique de proximité, contribue à la mise en place chez l'élève d'une conscience métalinguistique [...]. Par ce décloisonnement des sources d'apprentissage, l'élève se trouve encouragé à mettre en réseau différents savoirs et parvient à une meilleure conceptualisation grammaticale. [...] L'enrichissement du lexique se travaille en coordination avec les professeurs de français et de langues étrangères autour d'une sensibilisation aux problèmes de la traduction $»$.

18 Les langues régionales, dans ce nouveau cadre européen, doivent tirer parti de leur introduction relativement récente dans les enseignements du second degré et donc, libres de toute longue tradition didactique pesante, peuvent jouer un rôle de moteur dans l'adoption novatrice de la compétence plurilingue ${ }^{26}$ promue par le CECR. Leur participation à un enseignement bilingue organisé de la maternelle à la terminale les place de fait à l'avant - garde d'une didactique de la compétence plurilingue.

19 Nous avons déjà évoqué la particularité pédagogique des sections bilingues de langues régionales concernant les DNL : " aucune discipline ou aucun domaine disciplinaire, autre que la langue régionale, ne peut être enseigné exclusivement en langue régionale $»{ }^{27}$ Ce cadre légal qui instaure le bilinguisme dans l'enseignement des DNL invite à mettre en pratique un enseignement bilingue décloisonné. Il se définit par opposition à la juxtaposition de deux enseignements monolingues, l'un en français, l'autre en langue seconde, il permet le renforcement de la construction d'un même concept de DNL par le deuxième référent codique et symbolique, c'est à dire l'autre langue. Voici donc, au - delà du seul bénéfice linguistique et culturel, l'intérêt d'un enseignement bilingue décloisonné: la valeur ajoutée cognitive dont bénéficient les élèves dans les différentes disciplines. La construction pertinente des alternances linguistiques dans les séquences d'enseignement de langues et de DNL sera un facteur important de la réussite du bilinguisme décloisonné. Le décloisonnement linguistique au sein des DNL pourrait aussi ouvrir la voie, en particulier dans le second degré, à un décloisonnement entre les DNL se matérialisant par la pédagogie du projet pluridisciplinaire et donc aussi plurilingue.

$20 \mathrm{Au}$ - delà du bénéfice cognitif, l'enseignement bilingue décloisonné peut générer également un allègement curriculaire non négligeable. Les heures d'enseignement gagnées sur les redondances disciplinaires et linguistiques permettraient, au collège en particulier, d'éviter pour des élèves déjà bilingues le tiraillement entre les langues régionales, étrangères et anciennes et de mettre en place, au final, un décloisonnement des dispositifs à vocation linguistique en présence sur un même établissement. Les sections bilingues de langues régionales devraient pouvoir être combinées, pour les élèves qui le désirent, aux sections européennes ou orientales, aux parcours romans ou aux classes bi - langues, et inversement. Les sections bilingues ont vocation à devenir bi plurilingues, le bilinguisme n'étant qu'un cas particulier du plurilinguisme. 


\section{BIBLIOGRAPHIE}

BONET, L. (2007) : « Louis PASTRE : précurseur de l'enseignement intégré du catalan et du français, à l'école publique », in H. LIEUTARD \& M. - J. VERNY (éds.), L'école française et les langues régionales, actes du colloque CIRDOC des 13 et 14 octobre 2006, Publications de l'Université Paul Valéry, collection du CEO « Lo Gat Negre », Montpellier.

CAILLIS - BONET, F. (2007) : Quelles pratiques de classe pour un enseignement bilingue décloisonné ?, mémoire CAFIPEMF, Inspection Académique des Pyrénées - Orientales.

CAVALLI M. (2006) : «Créer une cohérence », in Le français dans le monde, n 344, mars / avril 2006, pp. $22-23$.

DUVERGER J. (2005) : L'enseignement en classe bilingue. Paris : Hachette.

GOULLIER, F. (2005) : Les outils du Conseil de l'Europe en classe de langue, Paris, Didier.

MALLART NAVARRA, J. (2006) : Multilingüisme \& educació (Textos docents ; 318), Publicacions i edicions de la Universitat de Barcelona.

MOORE, D. (2006) : Plurilinguismes et école, collection LAL (Langues et Apprentissage des Langues), Didier, Paris.

RUIZ BIKANDI U. (2005) : « La reflexió interlingüística : ajudar a pensar en / amb / sobre tres llengües » dans Articles, n 38, Barcelona, Graó.

\section{ANNEXES}

\section{Annexe 1}

Pyrénées-Orientales - Année scolaire 2005-2006

Effectifs* et évolution des formations bilingues franco-catalanes par niveau d'enseignement, enseignement public

\begin{tabular}{|l|c|c|c|c|c|c|c|c|}
\hline & $\begin{array}{c}\text { Répartition } \\
\text { des élèves } \\
\text { bilingues } \\
\text { par niveau }\end{array}$ & $\begin{array}{c}\text { Proportion } \\
\text { élèves } \\
\text { bilingues / } \\
\text { ensemble } \\
\text { des élèves } \\
\text { de catalan } \\
\text { de l'ensei- } \\
\text { gnement } \\
\text { public }\end{array}$ & $\begin{array}{c}\text { Proportion } \\
\text { élèves } \\
\text { bilingues / } \\
\text { ensemble } \\
\text { des élèves } \\
\text { de 1'ensei- } \\
\text { gnement } \\
\text { public }\end{array}$ & $\begin{array}{c}\text { Effectif } \\
\text { rentrée } \\
\mathbf{2 0 0 5}: \\
\text { élèves } \\
\text { bilingues } \\
\text { par niveau }\end{array}$ & $\begin{array}{c}\text { Variation } \\
\text { effectif } \\
\text { élèves } \\
\text { bilingues } \\
\text { rentrées } \\
\mathbf{2 0 0 5 / 2 0 0 0}\end{array}$ & $\begin{array}{c}\text { Nombre de } \\
\text { communes } \\
\text { avec des } \\
\text { classes } \\
\text { bilingues }\end{array}$ & $\begin{array}{c}\text { Nombre } \\
\text { d'établis- } \\
\text { sements } \\
\text { avec des } \\
\text { classes } \\
\text { bilingues }\end{array}$ & $\begin{array}{c}\text { Nombre de } \\
\text { classes ou } \\
\text { sections } \\
\text { bilingues }\end{array}$ \\
\hline Lycées & $\mathbf{5} \%$ & $19,8 \%$ & $1,1 \%$ & $\mathbf{8 2}$ & $+9,3 \%$ & 2 & 2 & 6 \\
Collèges & $\mathbf{1 2 , 8} \%$ & $11,8 \%$ & $1,3 \%$ & $\mathbf{2 1 0}$ & $+103,9 \%$ & 3 & 4 & 13 \\
Ecoles** & $\mathbf{8 2 , 2} \%$ & $14,3 \%$ & $3,7 \%$ & $\mathbf{1 3 4 9}$ & $+142,2 \%$ & 9 & 27 & 57 \\
\hline
\end{tabular}

* Selon données brutes du Rectorat de l'académie de Montpellier

** À partir d'une estimation des effectifs généraux du premier degré

Formations bilingues franco-catalanes publiques : 1641 élèves

- soit $2,7 \%$ des élèves de l'enseignement public des P.-O.

- soit $2,3 \%$ de l'ensemble des élèves des Pyrénées-Orientales

Pour mémoire : total enseignement du et en catalan dans les P.-O.

public + privé : 12777 élèves

dont 11160 public, soit $18 \%$ de l'ensemble

Annexe 2 


\section{Niveaux de compétences du Cadre Européen Commun de Référence et enseignement-apprentissage des Langues Vivantes Régionales*}

\begin{tabular}{|c|c|c|c|c|c|c|}
\hline & A1 & A2 & B1 & B2 & C1 & \\
\hline $\begin{array}{l}\text { Sections bilingues LVR, } \\
\text { parité horaire, à partir du cycle } 1^{(1)}\end{array}$ & $\begin{array}{c}\text { Cycles } \\
1 \text { et } 2\end{array}$ & $\begin{array}{c}\text { Cycle } \\
3\end{array}$ & $6^{\mathrm{e}}, 5^{\mathrm{e}}$ & $4^{\mathrm{e}}, 3^{\mathrm{e}}$ & $\begin{array}{l}2^{\mathrm{e}}, \\
1^{\mathrm{e}}, \\
\mathrm{T}^{\mathrm{e}}\end{array}$ & \\
\hline $\begin{array}{l}\text { LVR, } 1 \mathrm{~h} 30 \text { à } 3 \mathrm{~h}^{(2)} \\
\text { à partir du cycle } 1\end{array}$ & $\begin{array}{l}\text { Cycles } \\
1,2,3^{(3)}\end{array}$ & & & & & $L V 1^{(5)}$ \\
\hline $\begin{array}{l}\mathrm{LVR} / \mathrm{LV2}, 3 \mathrm{~h} \text {, à partir de la } 4^{\mathrm{e}} \text { et } \\
\text { poursuite d'étude à partir de la } 2^{\mathrm{e}} \text {. }\end{array}$ & & $4^{\mathrm{e}}, 3^{\mathrm{e}(4)}$ & $\begin{array}{l}2^{\mathrm{e}}, 1^{\mathrm{e}}, \\
\mathrm{T}^{\mathrm{e}}\end{array}$ & & & LV2 \\
\hline $\begin{array}{l}\text { LCR fac., } 2 \mathrm{~h} \text {, à partir de la } 6^{\mathrm{e}} \text { ou } \\
\text { LVR/LV3, 3h, à partir de la } 2^{\mathrm{e}}\end{array}$ & $\begin{array}{l}6^{\mathrm{e}}, 5^{\mathrm{e}} \\
4^{e}, 3^{e}\end{array}$ & $\begin{array}{l}2^{\mathrm{e}}, 1^{\mathrm{e}} \\
\mathrm{T}^{\mathrm{e}},\end{array}$ & & & & LV3 \\
\hline
\end{tabular}

* Dans tous les cas, l'enseignement d'une LV étrangère intervient en parallèle à la $L V$ régionale, dès le cycle 2.

(1) Les sections bilingues de langues régionales passent au niveau A2 dès le cycle 3, les LVE n'y accèdent qu'en sixième -- (2) Dans le cas du corse uniquement -- (3) Poursuite d'étude en LCR facultative en sixième, hors section bilingue -- (4) Élèves issus de cinquième LCR facultative -- (5) Les niveaux barrés d'une croix représentent la référence LV1 du CECR, pour les langues étrangères seulement. A l'exception des élèves du lycée Comte de Foix d'Andorre, pour le catalan, les langues régionales ne sont pas éligibles au titre de la LVl au baccalauréat.

Annexe 3

Comparaison plurilingue de la valeur (aspect) du passé simple et du passé composé, dans la langue orale

\begin{tabular}{|c|c|c|c|c|}
\hline Anglais & Francais & Occitan & Catalan & Espagnol \\
\hline $\begin{array}{l}\text { Simple past } \\
\text { He worked }\end{array}$ & $\begin{array}{c}\text { (Passé-simple) } \\
\text { (Il travailla) } \\
\text { ((vous travaillâtes)) } \\
\text { Passé-composé } \\
\text { Il a travaillé }\end{array}$ & $\begin{array}{c}\text { Preterit } \\
\text { Trabalhèt } \\
\\
\text { Passat compausat } \\
\text { A trabalhat }\end{array}$ & $\begin{array}{c}\text { Passat simple } \\
\text { (Pretèrit perfet } \\
\text { simple / sintètic) } \\
\text { Treballà } \\
\text { Passat perifràstic } \\
\text { (Pretèrit perfet } \\
\text { perifràstic) } \\
\text { Va treballar }\end{array}$ & $\begin{array}{c}\text { Pretérito indefinido } \\
\text { Trabajó }\end{array}$ \\
\hline $\begin{array}{l}\text { Present perfect } \\
\text { He has worked }\end{array}$ & & $\begin{array}{c}\text { Passat compausat } \\
\text { A trabalhat }\end{array}$ & $\begin{array}{c}\text { Perfet } \\
\text { (Pretèrit indefinit) } \\
\text { Ha treballat }\end{array}$ & $\begin{array}{c}\text { Pretérito perfecto } \\
\text { Ha trabajado }\end{array}$ \\
\hline
\end{tabular}

\section{NOTES}

1. La dichotomie langue étrangère versus langue régionale est toute relative : le français est langue régionale en Italie (Vallée d'Aoste, aux côtés du francoprovençal), l'allemand est langue régionale en France (Alsace, Moselle), l'occitan est co - officiel en Espagne (Vallée d'Aran), le catalan est officiel en Andorre, co - officiel en Espagne (Catalogne, Baléares, Valence) et protégé en Italie (Alghero en Sardaigne), etc.

2. Circulaire N 82 - 261 du 21 juin 1982 : 2) Ecole élémentaire [...] Enfin seront étudiées les conditions dans lesquelles pourraient être créées des classes expérimentales bilingues tenant compte des expériences déjà engagées dans certaines régions et faisant appel aux compétences qu'elles ont ainsi révélées.

3. Circulaire N 95 - 086 du 7 avril 1995 : Enseignement des langues et cultures régionales.

4. La loi N 94 - 665 du 4 août 1994, relative à l'emploi de la langue française, dite loi TOUBON, dispense de l'obligation d'enseigner exclusivement en français dans «les formations effectuées dans le cadre de l'enseignement des langues régionales ou étrangères [...] et représentant un maximum de $50 \%$ du volume total des enseignements ». 
5. Circulaire N 2001 - 167 du 05.09.2001 : Modalités de mise en oeuvre de l'enseignement bilingue à parité horaire.

6. Circulaire N 2003 - 090 du 05.06.2003 qui modifie la circulaire de 2001.

7. Arrêté du 12.05.2003, JO du 24.05.2003 : Enseignement bilingue en langues régionales à parité horaire dans les écoles et les sections « langues régionales » des collèges et des lycées. Texte paru consécutivement à l'annulation par le Conseil d'Etat, en 2002, d'une partie de l'appareil réglementaire sur l'enseignement des langues régionales qui faisait référence à la méthode de l'immersion totale.

8. Arrêté du 23.06.1994, JO du 26.06.1994 : Candidats des sections bilingues français - langue régionale.

9. Arrêté du 19 septembre 1991 : création de la section «Langue corse » et «Langue régionale » au CAPES. Le CAPES de langue corse est monovalent, les CAPES de langue régionale (basque, breton, catalan, créole, occitan - langue d'oc, tahitien) sont bivalents, dont l'histoire géographie (le français, l'anglais, l'espagnol) pour tous et les mathématiques uniquement pour le CAPES de breton et celui de tahitien. Les sections bilingues de collège en langue régionale existent actuellement en basque, breton, catalan, corse, occitan.

10. Décret $\mathrm{N}^{\circ} 2002$ - 11 du 3 janvier 2002 modifiant le décret $\mathrm{n}^{\circ} 90$ - 680 du 1er août 1990 relatif au statut particulier des professeurs des écoles et fixant les conditions dans lesquelles sont recrutés les professeurs des écoles chargés d'un enseignement de et en langue régionale.

11. www.emilangues.education.fr

12. www.flarep.com (Fédération pour les langues régionales dans l'enseignement public)

13. Annexe 1 : Effectifs et évolution des formations bilingues franco - catalanes, année scolaire 2005 - 2006.

14. www.crdp-montpellier.fr/languesregionales/

15. www.crdp-montpellier.fr/languesregionales/occitan/catalan_plaquette_bilingue.pdf

16. Loi $\mathrm{N} 51$ - 46 du 11 janvier 1951 : Enseignement des langues et dialectes locaux.

17. Loi N 2005 - 380 du 23.04.2005

18. Arrêté du 15.04.1988

19. Arrêté du 30 mai 2003.

20. Annexe 2 : Niveaux de compétence du CECR et langues régionales.

21. http://eduscol.education.fr/D0067/college-programmes.htm

22. Cadre Européen Commun de Référence pour les langues, Conseil de l'Europe, p. 129

23. GOULLIER, F . (2005), p. 105.

24. Ibidem

25. BO $n^{\circ} 6$ hors série du 25 août 2005.

26. Annexe 3: Comparaison plurilingue de la valeur (aspect) du passé simple et du passé composé, dans la langue orale.

27. Arrêté du 12.05.2003, JO du 24.05.2003 : Enseignement bilingue en langues régionales à parité horaire dans les écoles et les sections « langues régionales » des collèges et des lycées.

\section{RÉSUMÉS}

L'enseignement bilingue français - langue régionale, à parité horaire hebdomadaire dès la maternelle, diffère des sections européennes et de langues orientales. Dans les programmes de 
catalan, de corse et d'occitan, la compétence plurilingue du CECR trouve un écho favorable. Ce décloisonnement linguistique s'organise aux côtés d'un enseignement bilingue, réglementaire, des DNL. Les sections de langue régionale ont vocation à devenir bi - plurilingues avec l'inté gration précoce des langues étrangères.

The bilingual teaching of French - regional language, with an equal number of hours per week from nursery school onwards, differs from European and Oriental language departments. In the Catalan, Corsican and Occitan syllabuses the multilingual skills of the CEFR (Common European Framework of Reference for Languages) are viewed in a positive light. This linguistic decompartmentalization is set up alongside the statutory bilingual teaching of NLSs (non linguistic subjects). Regional language departments are destined to become bi - multilingual with the early integration of foreign languages.

\section{INDEX}

Mots-clés : bilinguisme, décloisonnement, Europe, français, langue régionale Keywords : bilingualism, decompartmentalization, French, regional language

\section{AUTEUR}

\section{LUC BONET}

Professeur de catalan en section bilingue, lycée Aristide Maillol de Perpignan et formateur associé à l'IUFM, site de Perpignan 